\title{
Fuzzy Counter-Ant for Avoiding the Stagnation of Multirobot Exploration
}

\author{
Abdelhak Chatty ${ }^{1,2}$, Ilhem Kallel ${ }^{1}$, Adel M. Alimi ${ }^{1}$, and Philippe Gaussier ${ }^{2}$ \\ ${ }^{1}$ REGIM: REsearch Group on Intelligent Machines \\ University of Sfax, National School of Engineers (ENIS), Sfax, Tunisia \\ \{abdelhak_chatty, ilhem.kallel, adel.alimi\}@ieee.org \\ ${ }^{2}$ ETIS ENSEA-UCP-CNRS 8051, University of Cergy-Pontoise, France \\ gaussier@ensea.fr
}

\begin{abstract}
Since swarm intelligence allows self-organization into an unfamiliar environment and adapting behaviors through simple individuals' interactions, we propose to realize a swarm multirobot organization with a fuzzy control. We introduce in this paper a fuzzy system for avoiding the collaboration stagnation and to improve the counter-ant algorithm (CAA). The robots' collaborative behavior is based on a hybrid approach combining the CAA and a fuzzy system learned by MAGAD-BFS (Multi-agent Genetic Algorithm for the Design of Beta Fuzzy System). A series of simulations enables us to discuss and validate both the effectiveness of the hybrid approach to the problem of environment exploration (i.e., for the purpose of cleaning an area) as well as the usefulness of MAGAD-BFS for learning the fuzzy knowledge base while tuning it and reducing its number of rules.
\end{abstract}

Keywords-multirobot, collaboration, pheromone, counter-ant algorithm, fuzzy system, MAGAD-BFS, stagnation recovery.

\section{INTRODUCTION}

The Ant algorithms provide powerful methods for the design of algorithms and optimization of distributed problems involving a collaborative swarm behavior [1-4]. Though the introduction of these algorithms into the world of robotics improves the communication quality between robots, the convergence time remains problematic [4-12].

Among swarm intelligence techniques in nature, the behaviors exhibited by ants are known to be among the most mature. In fact, the observation of ant colonies led to the so called ant colony algorithms [2, 5]. These revolve around a key concept called stigmergy which is an indirect coordination activity in an unknown environment: Ants are attracted to each other by a chemical material called pheromone. It seems that in an unknown environment, one of the most important problems related to multirobot systems is to decide how to coordinate actions in order to achieve tasks in an optimal way [8]. It is also very important to know what needs to be accomplished and the number of robots required for the task. In addition to this, the emergence of self-organizing cooperative behavior among the multirobot systems is noted. To formulate solutions that achieve self-organization, researchers resort to stigmergy. For example, when a robot finds out that it cannot achieve a mission, it marks the unfinished task with a quantity of pheromone. The more difficult the task is, greater the amount of pheromone used. Thus, robots will be tempted to try to accomplish their task [9].

It is clear that execution of a cargo pushing task is more natural if it is accomplished by a multirobot system using a cooperative ant colony strategy: In fact, when an ant tries to move cargo (e.g., food) alone, it spends moments to test the resistance of the cargo to forces applied by varying the orientation of the ant's body. Changing the direction of the applied strength can be enough to result in actual movement of the cargo. Should the realignment be inadequate, the ant releases the cargo and tries another position or direction from which to seize and move the cargo. If multiple adjustment attempts are not successful, the ant recruits other ants [8].

Many works such as those cited by Kube and Zhang [6][13] employ ant algorithms to solve object transportation problems. Other research consists of changing the ant algorithm using a new technique for multirobot pheromone placement. This technique enables robots to place more pheromones in the tasks that are about to be fulfilled: A very large amount of pheromone is placed on the cargo items that are close to successfully being moved or close to reaching their destinations, hence requiring a great force to move them. A small amount of pheromone is otherwise placed on the cargo. This strategy helps to attract additional robots to complete the task when needed [9]. In fact, the amount of pheromone is constant whereas in $[10,11]$ it is variable and depends on task difficulty and on the robots' strength. The problem of task allocation in the field of cooperative robotics is addressed by several studies especially in the context of unknown environments. Each robot has to adapt to its environment without any training stage. Moreover, the materialization of pheromone into real robots remains problematic in spite of the various attempts $[14,15]$.

In recent years, research has been oriented more towards the development of real-time systems and particularly the study of 
hybrid methods to ensure scalability of behavior with respect to the dynamic nature of the environment [16]. In [17], the authors present a hybrid path planning method, based on a counter ant algorithm and a fuzzy inference system, which enables multiple agents to find a solution rapidly along the unexplored regions.

To improve the uses of the ant algorithm, we present in this paper a solution based on fuzzy reasoning for the problem of stagnation behavior of evolving ant robots.

The remainder of this paper is organized as follows. Section II overviews the Counter-Ant algorithm. Sections III and IV present the proposal: a fuzzy system for the CAA referred to as FCA (Fuzzy Counter-Ant), and MAGAD-BFS for the design of the FCA in multirobot collaboration. Section V presents the simulation and discussion of the proposal. Finally, the last section concludes the paper.

\section{COUNTER-Ant Algorithm AS A PROPOSED COLLABORATION METHOD}

Let's consider another view of swarm intelligence inspired from ant colony optimization called Counter-Ant Algorithm (CAA) [17-19]. In reality, the robots' collaborative behavior is based on repulsion instead of attraction to pheromone- a chemical evaporating matter- that represents the core of ants' cooperation. The robots' reaction consists, henceforth, of avoiding paths covered by this chemical substance. The modified version includes a solution for stagnation recovery using pheromone positions.

The solution is then to give the ant the ability to become localized according to pheromone position: if its location is parallel to the pheromone, it has to change direction in order to find another way; otherwise, the presence of other ants in the same way is possible and may indicate a congested area. On the other hand, if its location is not parallel, it can move to avoid such potential. In this case, the ant does not avoid the pheromone since it does not have the same direction and we no longer refer to congestion areas. In simulation, we observe that the robots move into the environment by marking traveled paths with pheromone. During these random movements, robots apply a "path avoiding" stage. This strategy allows the ants to discover other regions not explored before, since paths marked by pheromone can divert them to new regions. Moreover, the long duration of pheromone evaporation makes the environment overloaded and limits robots' motion.

The random motion of robots in such an environment will limit the robots collaborative exploration, which affects the convergence time. The pure counter-ant is no longer a solution because robots will try to avoid paths of pheromone and as the environment is overloaded, then they will travel along the paths already marked out.

The novelty of the work presented in this paper is to optimize the random walk and uncertain robots and improve the quality of the collaborative exploration of the environment. The main step forward that this work represents relative to our previous work $[18,19]$ is to propose to integrate a fuzzy system inspired by the biological behavior of ants. If we observe the reaction of real ants in two different paths marked by pheromone (an experience for shortest path discovery), we note that ants react according to pheromone quantity in both paths and opt for the path containing the highest pheromone quantity.

Figure 1 shows that the black ant thinks about the pheromone quantity on the path divergence. It will take the path having the highest pheromone quantity that represents the shortest path. In the next section, we try to integrate this idea with the fuzzy system.

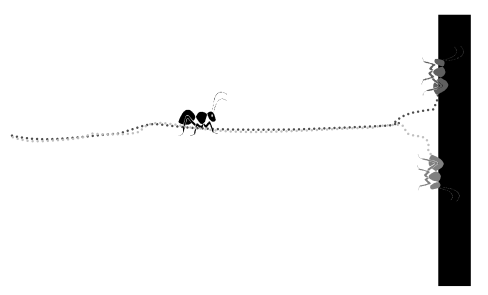

Figure 1. Path selection based on pheromone quantity.

\section{FUZZY SYSTEM FOR AVOIDING THE COLlABORATIVE STAGNATION}

In order to improve the convergence of the counter ant algorithm and random motion of robots, we propose to integrate a fuzzy rule based system and apply it to a collaborative cleaning task.

\section{A. Analyzing the Situation}

Fig. 1 shows the black ant while choosing an adequate path. By applying the counter ant algorithm, this ant will maintain its trip on the black path until it notices that its position is confused with this path. In this case, it changes its direction randomly, which negatively affects the environment exploration quality. However, if we hedge a bit in the ant reasoning method, we can have better results. The ant does not think about observed pheromone quantity as on the number of paths marked on its front, left, or right (see Fig. 2). Therefore, these three factors will allow the ant to decide the rotation angle and the speed of its next motion.

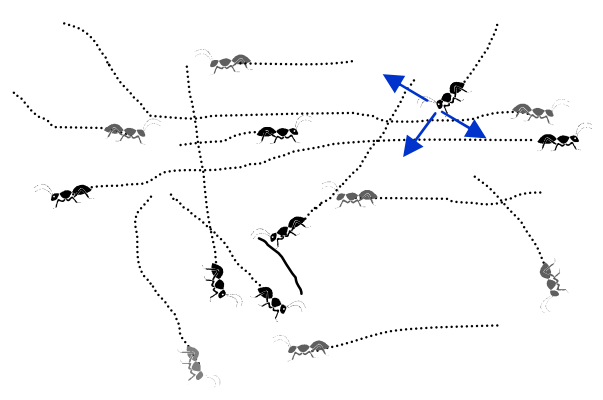

Figure 2. Detection of the number of marked paths in the three regions.

In order to solve such situations, fuzzy logic [20] proves to be suitable [21]. This approach uses inputs and a fuzzy 
inference system to generate, in this case, two outputs corresponding to the rotation angle and motion speed. The fuzzy system, when it is well designed, will have to solve the problem of collaborative robots' stagnation behavior and, therefore, to improve robots motion quality since they can exploit more new paths; this can minimize the total exploration time.

Fig. 3 presents the integration of a fuzzy system in the CAA. This integration creates a change in the behavior of the robots with respect to saturation of the environment via the pheromone traces.

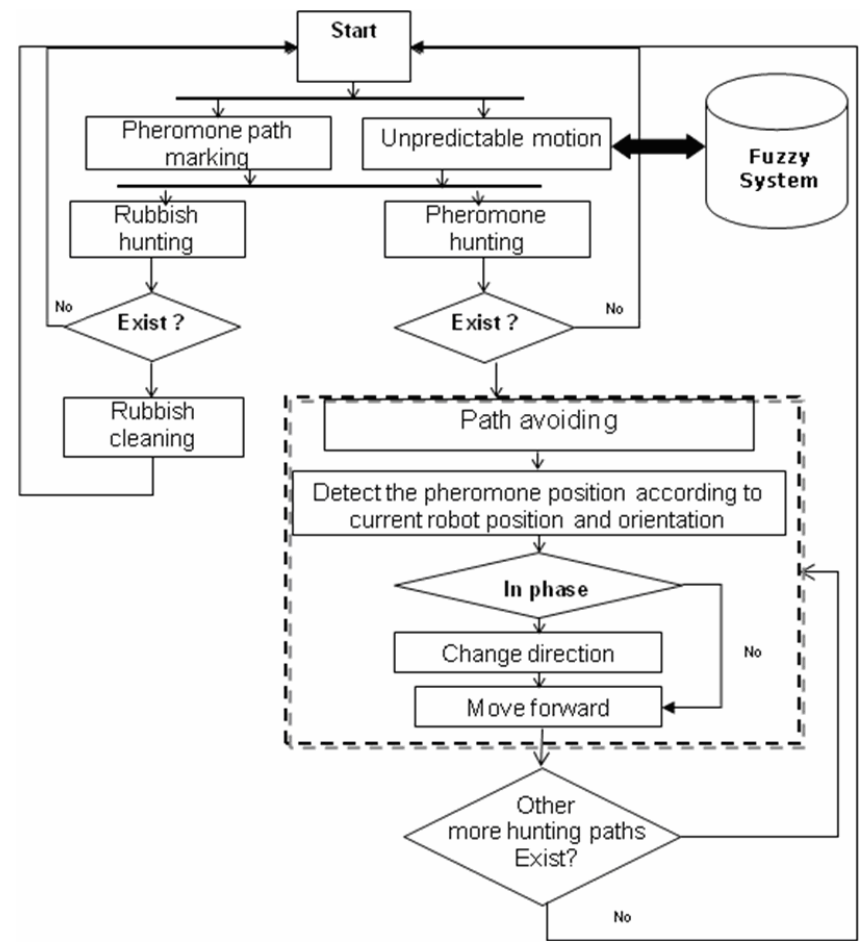

Figure 3. The flowchart of the hybrid CAA-fuzzy system.

These behaviors can be classified into three phases:

(i) CAA phase: Ants begin by moving randomly to hunt rubbish in the ant-hill, which presents an unknown environment in our proposal. In this unknown environment may consist of rubbish. While ants move they mark out their paths by pheromone, in other words, they begin to secrete pheromone for fear of being followed by other ants. The presence of this substance indicates the paths which are already traveled by other ants. In order to ensure a collaborative cleaning task, ants avoid these paths and circumvent blocked cleaning zones by changing motion direction according to the position of pheromone. The long duration of the evaporation of traces of pheromones increases the number of paths in the environment. This coincides with the following phase (ii).

(ii) Hybrid system phase: We have increased our interest in optimizing the CAA to improve the quality of the exploration of the environment due to random movements of the robots. We propose a fuzzy system which can generate ants' speeds and their angles of rotation. It is important to note that the pheromone is both the basis of our CAA and that of FCA since its inputs are based on the pheromone. This hybrid method can solve the stagnation problem of collaborative robots, and then accelerate the convergence time. So, the robots move using the CAA and when detecting multiple paths, they operate their fuzzy system to determine the direction and speed to avoid areas already exploited by other robots.

Finally, (iii) Fuzzy system phase: persistence of pheromone traces creates an overloaded environment so each robot applies apply the fuzzy system to exploit new areas without passing through areas covered by other robots.

\section{B. Architecture of the Proposed Fuzzy System}

A Takagi Sugeno (TSK) fuzzy system is applied [22]. We don't use the regular Mamdani rules wich vave proven to be sufficient in many prior applications of fuzzy logic-based mobile robot navigation because the TSK fuzzy system gives concrete output values without need of defuzzification phase. Fig. 4 illustrates the architecture of the proposed fuzzy system having three inputs: the number of marked paths on the left of the robot, on its front and on its right; and two outputs: the rotation angle and the motion speed.

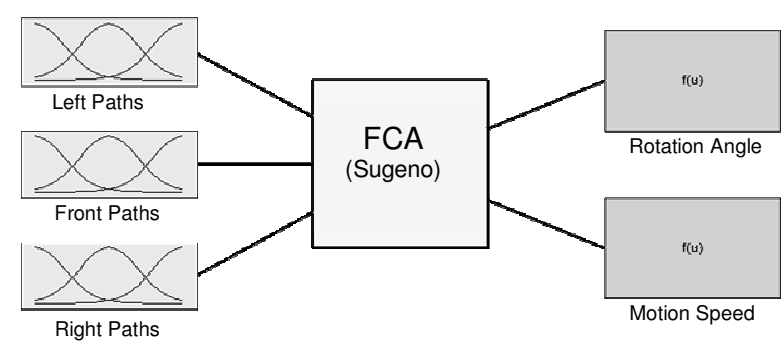

Figure 4. Architecture of the proposed fuzzy system.

1) Initial data base: Initially, we adopt as membership functions both trapezoidal and triangular functions. All input variables are defined by three fuzzy subsets (small, middle and big). Mathematically, these functions are defined respectively by equations (1), (2) and (3).

$$
\begin{gathered}
\mu_{\text {Small }}(x)=\left\{\begin{array}{cc}
1 & \text { if } 1 \leq x \leq \frac{3}{2} \\
\frac{4}{3}-\frac{2}{9} x & \text { if } \frac{3}{2}<x \leq 6
\end{array}\right. \\
\mu_{\text {M iddle }}(x)=\left\{\begin{array}{cc}
\frac{2}{9} x-\frac{1}{3} & \text { if } \frac{3}{2} \leq x<6 \\
1 & \text { if } x=6 \\
4-\frac{1}{2} x & \text { if } 6<x \leq 8
\end{array}\right. \\
\mu_{\mathrm{Big}}(x)=\left\{\begin{array}{cc}
\frac{1}{2} x-3 & \text { if } 6 \leq x<8 \\
1 & \text { if } x \geq 8
\end{array}\right.
\end{gathered}
$$


The outputs are:

Rotation angle: The change in direction degree according to the robot orientation;

Motion speed: The velocity value $1 \mathrm{~mm} / \mathrm{s}$ of the robot in the next motion.

2) Initial rule base: In fuzzy systems, rules express the relationship between input variables as linguistic variables and output variables. Since we do not have any automatic benchmark, we have to design, manually, an initial rule base (see Table I) and then generate an example of bases according to real motion and behaviors. It should be noted that the following rules presented all possible cases after series of tests.

TABLE I. INTIAL RULE BASE FOR COLLABORATIVE BEHAVIOR ENHANCEMENT

\begin{tabular}{|c|c|c|c|c|c|}
\hline $\begin{array}{l}\text { Rule } \\
\text { number }\end{array}$ & Front.Paths & Left.Paths & Right.Paths & $\alpha \circ$ & $\begin{array}{l}v_{1} \\
m m / \\
s\end{array}$ \\
\hline 1 & Small & small & Small & 15 & 12 \\
\hline 2 & Small & small & middle & 17 & 11 \\
\hline 3 & Small & small & Big & 20 & 10 \\
\hline 4 & Small & middle & small & 340 & 6 \\
\hline 5 & Small & middle & middle & 350 & 4 \\
\hline 6 & Small & middle & Big & 18 & 5 \\
\hline 7 & Small & big & small & 0 & 9 \\
\hline 8 & Small & big & middle & 345 & 3 \\
\hline 9 & Small & big & Big & 1 & 2 \\
\hline 10 & Middle & small & small & 45 & 8 \\
\hline 11 & Middle & small & middle & 50 & 7 \\
\hline 12 & Middle & small & Big & 55 & 6 \\
\hline 13 & Middle & middle & small & 310 & 5 \\
\hline 14 & middle & middle & middle & 320 & 4 \\
\hline 15 & middle & middle & big & 60 & 3 \\
\hline 16 & middle & big & small & 330 & 9 \\
\hline 17 & middle & big & middle & 2 & 4 \\
\hline 18 & middle & big & big & 120 & 8 \\
\hline 19 & big & small & small & 90 & 7 \\
\hline 20 & big & small & middle & 93 & 6 \\
\hline 21 & big & small & big & 95 & 8 \\
\hline 22 & big & middle & small & 270 & 7 \\
\hline 23 & big & middle & middle & 275 & 3 \\
\hline 24 & big & middle & big & 92 & 2 \\
\hline 25 & big & big & small & 273 & 6 \\
\hline 26 & big & big & middle & 276 & 2 \\
\hline 27 & big & Big & big & 220 & 8 \\
\hline
\end{tabular}

3) Computation of the Sugeno Output: The computation of the global system output according to the weighted average is among the most accurate method and it is formulated by (4).

$$
\text { output }=\frac{\sum_{\mathrm{i}=1}^{\mathrm{n}} W_{\mathrm{i}} \times Z_{\mathrm{i}}}{\sum_{i=1}^{n} W_{\mathrm{i}}}
$$

Where $W_{\mathrm{i}}$ and $Z_{\mathrm{i}}$ are the activation degree and the output of the rule $i$ respectively, and $n$ is the number of rules.

By applying this equation on the fuzzy counter-ant system, we can compute the rotation angle $\alpha$ and the motion speed $v$.

\section{MAGAD-BFS FOR THE DESIGN OF THE FCA SYSTEM}

The MAGAD-BFS (Multi-agent Genetic Algorithm in the Design of Beta Fuzzy System) method [23] is used for the learning and design of the fuzzy counter-ant system knowledge base. The objective of $M A G A D-B F S$ is to find the optimal fuzzy system structure and its different parameters while reducing the rule complexity and avoiding a genetic local optimum.

The main idea of $M A G A D-B F S$ is to produce sub-populations according to a genetic terminology detailed in [23]. Assigned to worker agents, each sub-population consists of chromosomes having the same size (i.e. the same number of fuzzy rules). This approach involves a number of agents continually in interaction that can mutate during the general process, in order to find the best knowledge base, which is the best chromosome, while considering a compromise between the precision of the system output and its lowest number of rules. Each worker agent executes its local GA, communicates its best solution to a catalyst agent which executes a GA in order to take out again the best of them. The obtained chromosome will be injected in population of a worker agent having the same chromosome length, if exists. Otherwise it will be injected in another agent, judged non efficient and, therefore, intended for mutation. defined by equation (5), as fuzzy basis function does not require normalization as in classical fuzzy logic system. Thus, activation intensity of each rule is characterized by one Beta function [24].

$$
\beta\left(x, x_{c}, D, p, q\right)=\left\{\begin{array}{cc}
{\left[1+\frac{p+q}{D p}\left(x-x_{c}\right)\right]^{p}\left[1+\frac{p+q}{D q}\left(x_{c}-x\right)\right]^{q}} \\
\text { if } x \in\left[x_{c}-\frac{D p}{p+q},\right. & \left.x_{c}+\frac{D q}{p+q}\right] \\
0 & \text { elsewhere }
\end{array}\right.
$$

Where $x=\left(x_{1}, x_{2}, \ldots, x_{n}\right)^{T}$ is the input variable ; $X_{c}$ and $D$ are respectively the kernel and the width (carrier) of the Beta function ; $p>0$ and $q>0$, are real parameters.

For the training task of all fuzzy logic units we use the parameter values detailed in Table II and Table III.

TABLE II. GA TRAINING PARAMETERS OF MAGAD-BFS FOR FCA

\begin{tabular}{lll}
\hline \hline GA Parameters & Catalyst & Worker Group \\
\hline Maximum number of generations & 1000 & 1000 \\
Initial population size & 80 & 80 \\
Evolution rate of population size & $100 \%$ & $100 \%$ \\
Crossover probability “Column-cross" & 0.7 & 0.7 \\
Crossover probability “Mask-cross" & 0.00001 & 0.00001 \\
Mutation probability & 0.001 & 0.001 \\
permutation probability & 0.001 & 0.001 \\
Addition probability & 0.001 & ----- \\
Elimination probability & 0.001 & ---- \\
\hline \hline
\end{tabular}


TABLE III. INITIAL PARAMETERS OF MAGAD-BFS FOR FCA

\begin{tabular}{|c|c|c|c|c|}
\hline & Parameters & $\begin{array}{l}\text { Number } \\
\text { of paths }\end{array}$ & $\begin{array}{l}\text { Rotation } \\
\text { Angle }(\alpha)\end{array}$ & $\begin{array}{l}\text { Motion } \\
\text { Speed }\end{array}$ \\
\hline \multirow{6}{*}{$\begin{array}{l}\text { Training } \\
\text { Parameters }\end{array}$} & Domain & {$[0,100]$} & {$[15,365]$} & {$[2,10]$} \\
\hline & $\begin{array}{l}\text { Number of } \\
\text { training data }\end{array}$ & 12117 & & \\
\hline & Domain of $x_{c}$ & {$[0,100]$} & {$[15,365]$} & ---- \\
\hline & Domain of D & {$[5,30]$} & {$[0.05,60]$} & ----- \\
\hline & Domain of $\mathrm{p}$ & {$[0.1,1.8]$} & {$[0.1,1.8]$} & ---- \\
\hline & Domain of $q$ & {$[0.1,1.8]$} & {$[0.1,1.8]$} & ----- \\
\hline Initial & $\begin{array}{l}\text { Minimum } \\
\text { number of rules }\end{array}$ & 4 & & \\
\hline agents & $\begin{array}{l}\text { Maximum } \\
\text { number of rules }\end{array}$ & 27 & & \\
\hline
\end{tabular}

The training phase of the entire knowledge base defining the proposed fuzzy CAA gives us many solutions as best knowledge base. We have given just one of them according to the best tradeoff between precision and number of rules. Fig. 5 presents the Beta membership functions of the 8 fuzzy subsets defining input variables, and Fig. 6 presents the 8 Beta fuzzy rules designing the fuzzy CAA behavior.
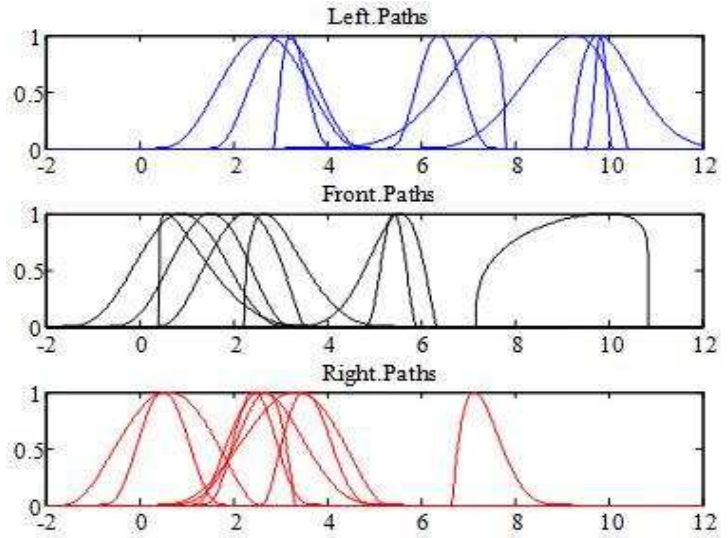

Figure 5. Beta membership functions of the 8 fuzzy subsets defining input variables.
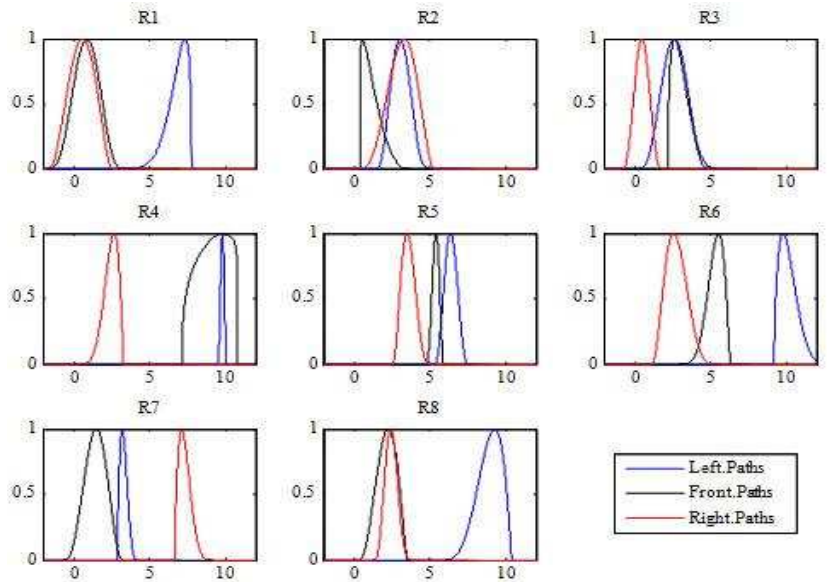

Figure 6 . The 8 Beta fuzzy rules designing the F CA behavior.

\section{SimUlations AND DiscUSSION}

After research in the cleaning field, we notice that cleaning of an unknown environment by a multirobot system conceals some complexity in terms of inter-robot coordination and efficiency. In fact, we notice a congestion in the cleaning zones, which negatively influences the convergence time. To better discuss this function, we implement the FCA in the simulation platform Madkit ${ }^{1}$.

Collaboration between robots is done in a completely unknown environment. It is made up mainly by objects and cleaning robots, which are implemented and generated using with simulation platform. These cleaning robots are provided a sensor enabling them to detect the pheromone trace.

\section{A. Influence of the fuzzy system on the Counter-Ant}

The simulation contains 5 robots and 80 rubbish items. The scope of perception is $100 \mathrm{~mm}$ and the time of evaporation of pheromone is 1320 s. Fig. 7 shows the end of the cleaning operation after $t=59.61 \mathrm{~s}$ using the hybrid system (CAA and FCA).

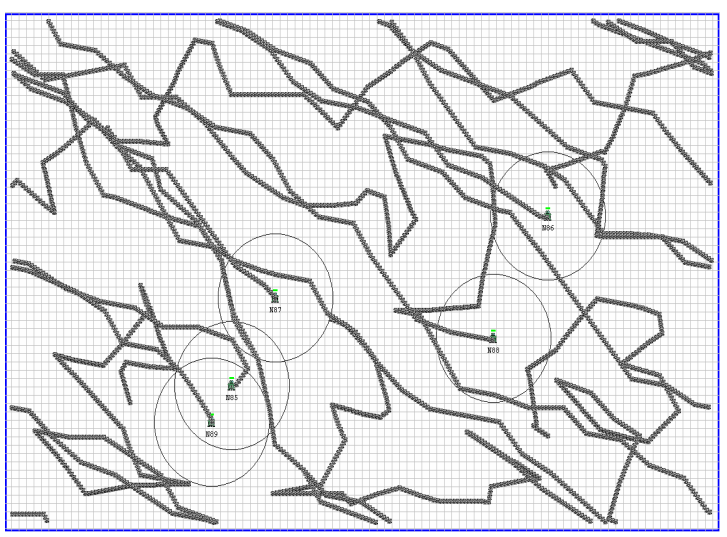

Figure 7. The end of the cleaning operation using the hybrid method at $\mathrm{t}=59.61 \mathrm{~s}$.

To show that the fuzzy system allows agents to improve the collaborative exploration and random movements, we try to analyze the agents' behaviors in an environment overloaded by the pheromone.

Fig. 8a shows that agents are in an overloaded area. At time $151.32 \mathrm{~s}$, the agents can exceed this area to another not yet explored (see Fig. 8b). Here, agents currently apply the $\mathrm{CAA}$, and hence avoid the congestion of areas.

Fig. 8c and Fig. 8d show that robots are able to move liberally when applying the counter-Ant algorithm and the fuzzy system. This sums up the impact of the hybrid method for inter-robot cooperation, overcoming stagnation and the collaborative explored environment.

Fig. 8 e shows that at time $t=613.33 \mathrm{~s}$, robots continue to explore collectively the remaining undiscovered areas by applying the hybrid approach, despite the overloaded environment.

\footnotetext{
${ }^{1}$ http://www.madkit.org
} 


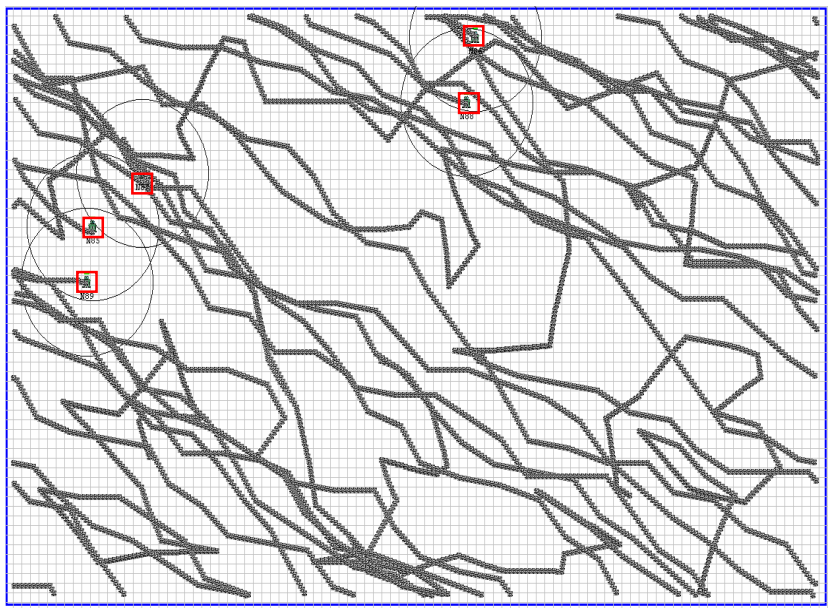

(a) $\mathrm{t}=150.79 \mathrm{~s}$

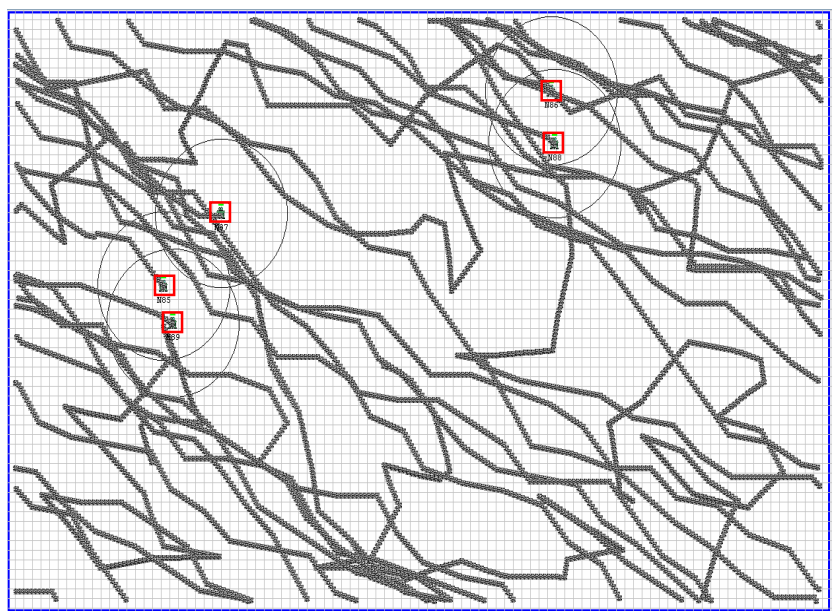

(b) $\mathrm{t}=151.32 \mathrm{~s}$

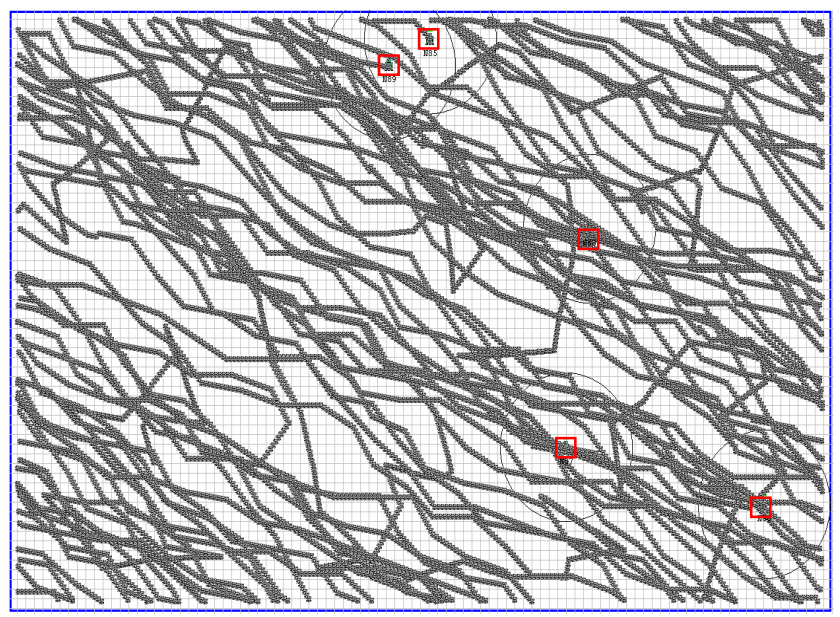

(c) $\mathrm{t}=550.08 \mathrm{~s}$

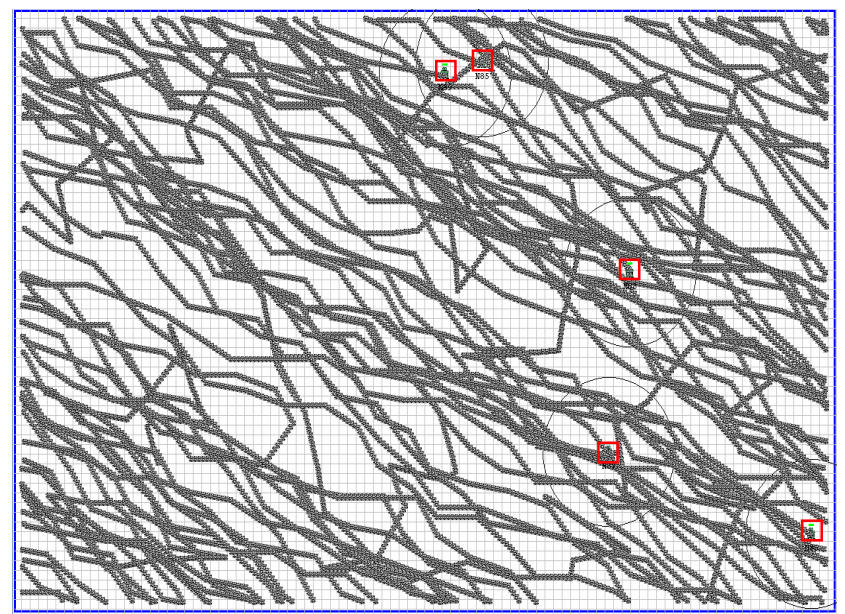

(d) $\mathrm{t}=55.80 \mathrm{~s}$

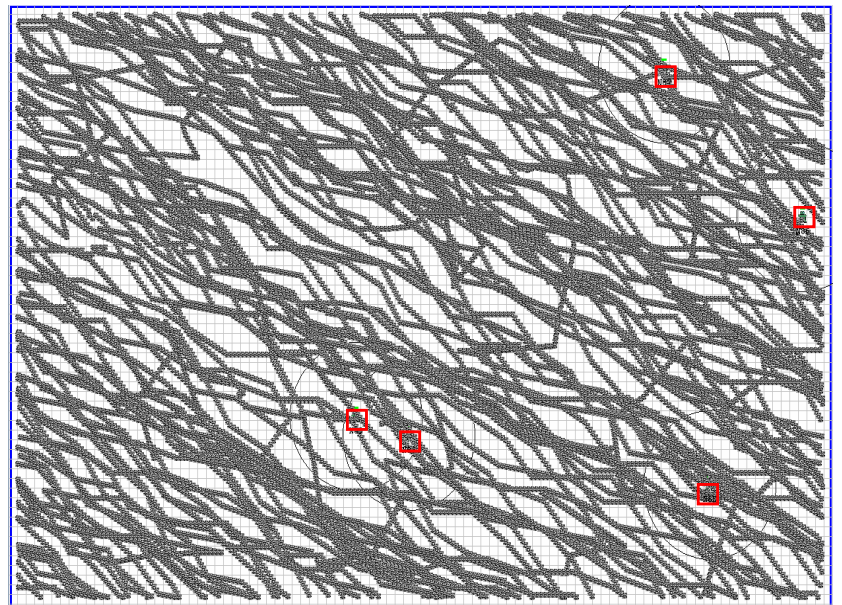

(e) $\mathrm{t}=613.33 \mathrm{~s}$

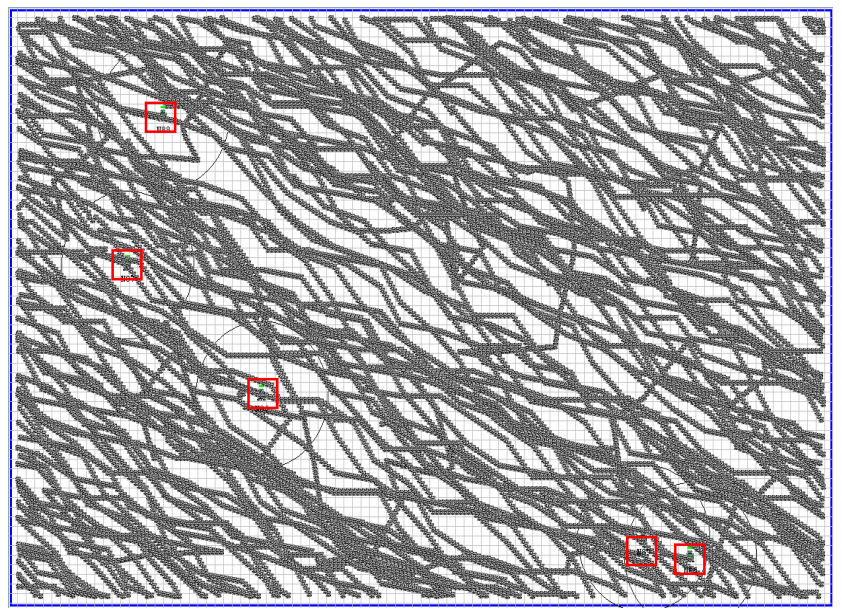

(f) $\mathrm{t}=754.30 \mathrm{~s}$ 
Fig. 8f shows that the robots are scattered in the environment thanks to the application of the fuzzy system which avoids the stagnation of the exploration.

We attest in [18] that the persistence of the pheromone trace for a long life increases the convergence time because the environment will be encumbered, which limits the robot motion.

Fig. 9 shows that despite the clutter of the environment through pheromone traces, robot N85 continues to explore the remaining undiscovered areas. In fact, the fuzzy system allows to optimize the random motion of robots by avoiding the stagnation behavior. This leads to improve the quality of the collaborative exploration of the environment.

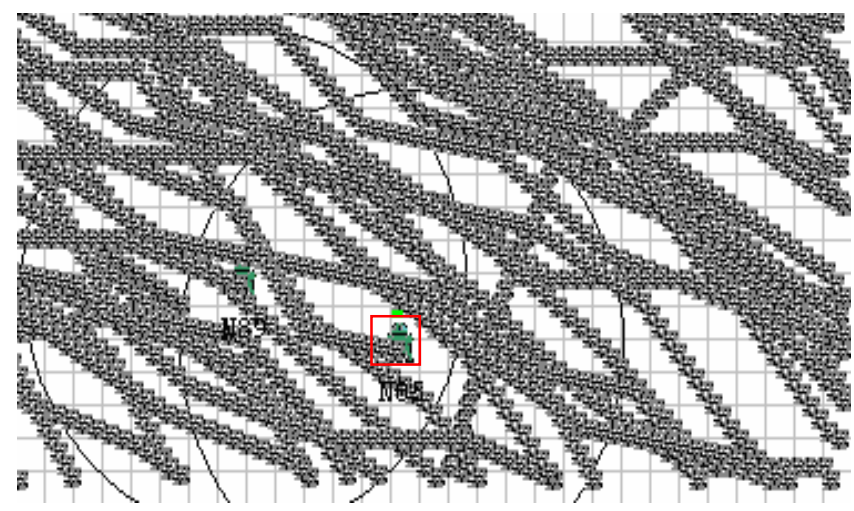

Figure 9. Zoom of the robot neighborhood

Based on tests of the hybrid method, FCA, we consider it valid to solve the problem of environment exploration, including cleaning an area and facilitating robots' collaboration. It is important to note that FCA is considered useful for providing basic local rules to create emergent phenomena in an embedded multirobot system.

In a few encountered cases, the priority assigned to the cleaning task generates competitive behavior among robots: upon detection of rubbish the robot moves toward it to clean it; but in the presence of another robot (10a), it performs both the same behavior to clean the rubbish (10b).

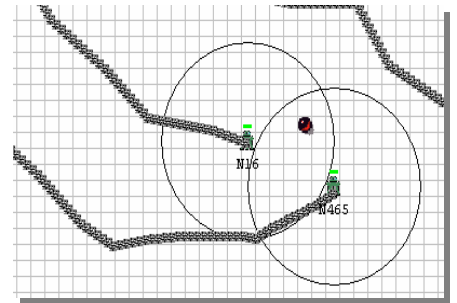

(a)

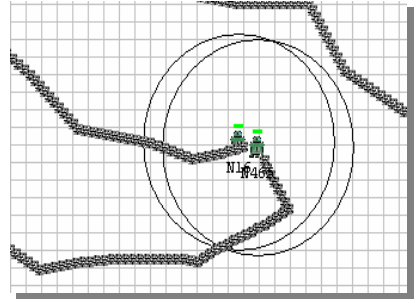

(b)
Figure 10. Competitive behavior.

If we contemplate the pheromone traces in Fig. 11, we note that there is an emerging diagonal trend of robot trails. So, the question is, can we control such an emerging property and create others?

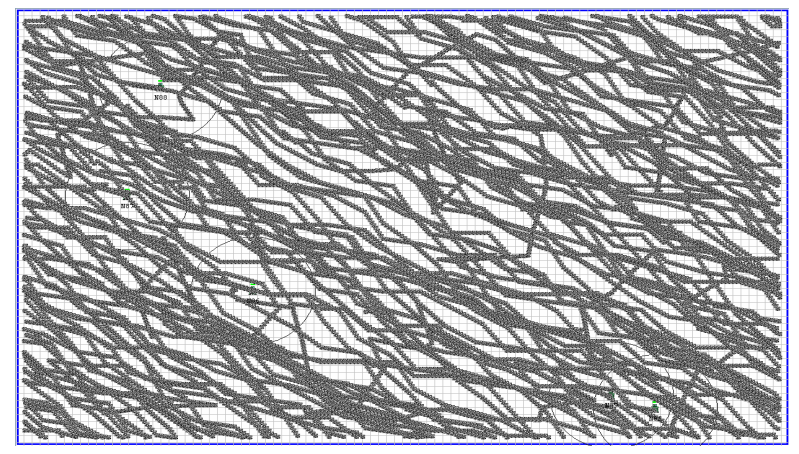

Figure 11. Emerging direction.

\section{B. Alliance: the co-operative control Vs the hybrid system} FCA

The Alliance architecture suggested by Parker [25] based on faults tolerance is a supervised architecture which consists on collaborating robots to clean two sources of rubbish. The communication between robots is done through the diffusion of messages about sources localization via a supervision system. Alliance is certainly based on the faults tolerance however, the supervision of the robots present in itself a limit of the solution. It is important to mention that the quality of the exploration of the environment was not approached. Alliance presents a specific solution for rubbish cleaning gathered and not scattered. However, our FCA can be well applied to scattered rubbish cleaning in unknown environment. It is an unsupervised solution with "stigmergy" as the base of communication and collaboration. In fact, it possible to solve the cleaning problem while taking into account the quality of the dilemma "convergence time/goal to reach". Also we optimize the random walk and uncertain robots and improve the quality of the collaborative exploration of the environment. Table IV lists the main features of the proposed hybrid system, FCA.

TABLE IV. REVIEW OF SOME FEATURES IN FCA VS ALLIANCE

\begin{tabular}{|c|c|c|}
\hline Approach & Hybrid fuzzy/ant (FCA) & Alliance [25] \\
\hline Supervision architecture & No & Yes \\
\hline Communication Method & Stigmergy & Sensors \\
\hline Communication tool & Pheromone & $\begin{array}{l}\text { Diffusion of } \\
\text { messages }\end{array}$ \\
\hline Fault tolerance & Yes & Yes \\
\hline $\begin{array}{l}\text { Exploration of } \\
\text { environment }\end{array}$ & CAA & - \\
\hline $\begin{array}{l}\text { Exploration of } \\
\text { overloaded environment }\end{array}$ & FCA & - \\
\hline Avoiding stagnation & Hybrid system & - \\
\hline Application & $\begin{array}{l}\text { Exploration and } \\
\text { Cleaning }\end{array}$ & Cleaning \\
\hline Convergence time & Adequate & Long \\
\hline
\end{tabular}




\section{CONCLUDING REMARKS}

In this paper, we propose to realize an improved swarm multirobot organization with a fuzzy control. It is a hybrid and cooperative model to solve the problem of collaborative robots stagnation behavior evoked in previous work [18]. We propose here to integrate a fuzzy rule based system according to the number of marked paths on the left and right and at the front of the robot. An implementation followed by a series of simulations allowed us to obtain an example base in order to perform the off-line learning by MAGAD-BFS [23]. This enables us to discuss and validate both the effectiveness of hybrid and collective method for the problem of environment exploration i.e. area cleaning, as well as the usefulness of $M A G A D-B F S$ for learning the knowledge base while reducing the rule base and tuning the data base.

\section{ACKNOWLEDGMENT}

The authors thank Professor Georgi Dimirovski for his advises. They also acknowledge the financial support of this work by grants from General Direction of Scientific Research (DGRST), Tunisia, under the ARUB program.

\section{REFERENCES}

[1] M.Dorigo and G. DiCaro, "The Ant Colony Optimization MetaHeuristic", New Ideas in Optimization 1999. (New York: McGrawHill)

[2] M.Dorigo and T. Stützle, "Ant colony optimization". Cambridge, MA, USA: The MIT Press, 2004

[3] H.Szu, P. Chanyagorn, W. Hwang, M. Paulin and T. Yamakawa, "Collective and distributive swarm intelligence: evolutional biological survey", International Congress Series, Volume 1269, August 2004, Pages 46-49

[4] M.Mucientes and J.Casillas, "Quick design of fuzzy controllers with good interpretability in mobile robotics", in IEEE Trans. on Fuzzy Systems, vol. 15, no. 4, 2007, pp. 636-651.

[5] G.Dudek, M. Jenkin, E. Milios and D. Wilkes, "A taxonomy for swarm robots", Intelligent Robots and Systems, IROS '93. Proc. of the 1993 IEEE/RSJ, , Vol. 1, July 1993, pp. 441-447.

[6] C.R.Kube, C.R. and H. Zhang, "Collective robotics: From social insects to robots", Adaptive Behavior 2, 189-218, 1994

[7] O.Holland and C. Melhuish, Stigmergy, "self-organization, and sorting in collective robotics", Artificial Life, vol. 5, no. 2, 1999, pp. 173-202.

[8] C.R.Kube and E. Bonabeau, "Cooperative transport by ants and robots," Robotics and Autonomous Systems, vol. 30, 2000, pp. 85-101.

[9] D.Yingying, H. Yan, J. Jingping, "Multi-Robot Cooperation Method Based On The Ant Algorithm”, Proc. of the IEEE, 2003.

[10] E.Borzello and L. D. Merkle, Multi-Robot "Cooperation Using the Ant Algorithm with Variable Pheromone Placement", Proceedings of the IEEE, 2005
[11] D.Zhang, G. Xie, J.i Yu, and L. Wang, “Adaptive task assignment for multiple mobile robots via swarm intelligence approach," Robotics and Autonomous Systems, vol. 55, no. 7, 2007, pp. 572-588

[12] M.Mamei and F.Zambonelli, "Pervasive Pheromone-Based Interaction with RFID Tags", in ACM Trans. on Autonomous and Adaptive Systems, vol. 2, 2007, pp. 1-28.

[13] C.R.Kube and H. Zhang, "Stagnation recovery behaviors for collective robotics", in: Proceedings of the 1994 IEEE/RSJ/GI International Conference on Intelligent Robots and Systems, IEEE Computer Society Press, Los Alamitos, CA, 1995, pp. 1883-1890.

[14] A.H.Purnamadjaja and R.A Russell, "Pheromone communication: implementation of necrophoric bee behaviour in a robot swarm", Robotics, Automation and Mechatronics, 2004 IEEE, Dec. 2004, Vol. 2, pp.638- 643.

[15] A.H.Purnamadjaja and R.A. Russell, "Robotic Pheromones: Using Temperature Modulation in Tin Oxide Gas Sensor to Differentiate Swarm's Behaviours", in 9th Int. Conf. on Control, Automation, Robotics and Vision ICARCV, 2006, pp. 1-6.

[16] P. Angelov, R. Buswell, "Identification of Evolving Rule-based Models", IEEE Transactions on Fuzzy Systems, ISSN 1063-6706, vol. 10, No5, pp. 667-677, 2002

[17] M.Ahuja, B.HomChaudhuri, K. Cohen and M. Kumar, "Fuzzy counter ant algorithm for maze problem", 48 ${ }^{\text {th }}$ AIAAA Aerospace Science Meeting Including the New Horizons Froum and Aerospace Exposition, Orlando, FL, Paper IAAA 2010-595, January 2010

[18] A.Chatty, I. Kallel, and A.M. Alimi, "Counter-Ant Algorithm for Evolving Multirobot Collaboration. Application to Collaborative Cleanup", in Proc. of the Int. Conf. on Soft Computing as Transdisciplinary Science and Technology IEEE/ACM CSTST, Paris, 2008, pp. 84-89

[19] I.Kallel, A. Chatty, and A.M. Alimi A.M., "Self-organizing Multirobot Exploration through Counter-Ant Algorithm", in Proc. of the 3rd Int. Workshop on Self-Organizing Systems IWSOS, Lecture Notes in Computer Science (LNCS) series, vol. 5343, 2008, pp. 133-144.

[20] L.A.Zadeh, "Fuzzy sets", in Information Control, vol. 8, 1965, pp. 338 358

[21] J.Casillas, O. Cordón, I. Fernández de Viana, F. Herrera, "Learning cooperative linguistic fuzzy rules using the best-worst ant system algorithm", International Journal of Intelligent Systems 20:4, 2005.

[22] M.Sugeno and G.T. Kang, "Structure Identification of Fuzzy Models", in Fuzzy Sets and Systems, vol. 28, 1988, pp. 15-33

[23] I.Kallel and A.M. Alimi, "MAGAD-BFS: A Learning Method for Beta Fuzzy Systems based on a Multi-Agent Genetic Algorithm", in Int. J. of Soft Computing, A Fusion of Foundations, Methodologies and Applications, Focus: New Trends in the Fuzzy Modelling Part I: Novel Approaches, Springer-Verlag, vol. 10, no. 9, 2006, pp. 757-772.

[24] A.M. Alimi, "The Beta-Based Neuro-Fuzzy System: Genesis and Main Properties", in TASK Quarterly J., Special Issue on Neural Networks, W. Duch and D. Rutkowska (Eds.), vol. 7, no. 1, 2003, pp.23-41.

[25] L.Parker "ALLIANCE: An Architecture for Fault Tolerant Multi-Robot Cooperation," IEEE Transactions on Robotics and Automation, vol. 14, no. 2, 1998, pp.220-240. 